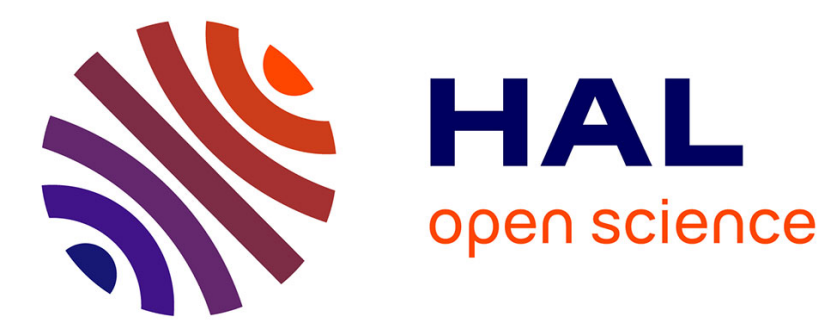

\title{
Constant-angle surfaces in liquid crystals
}

Paolo Cermelli, Antonio J. Di Scala

\section{- To cite this version:}

Paolo Cermelli, Antonio J. Di Scala. Constant-angle surfaces in liquid crystals. Philosophical Magazine, 2007, 87 (12), pp.1871-1888. 10.1080/14786430601110364 . hal-00513811

\section{HAL Id: hal-00513811 \\ https://hal.science/hal-00513811}

Submitted on 1 Sep 2010

HAL is a multi-disciplinary open access archive for the deposit and dissemination of scientific research documents, whether they are published or not. The documents may come from teaching and research institutions in France or abroad, or from public or private research centers.
L'archive ouverte pluridisciplinaire HAL, est destinée au dépôt et à la diffusion de documents scientifiques de niveau recherche, publiés ou non, émanant des établissements d'enseignement et de recherche français ou étrangers, des laboratoires publics ou privés. 


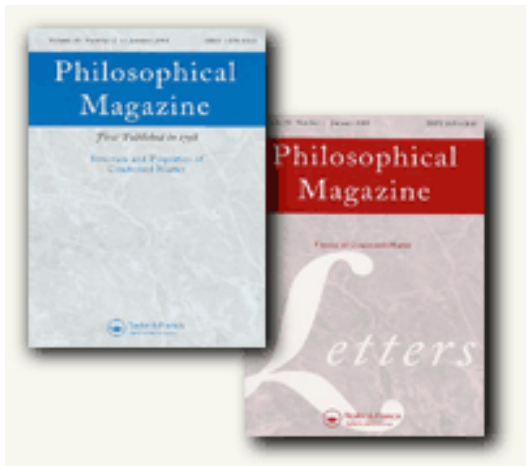

\section{Constant-angle surfaces in liquid crystals}

\begin{tabular}{|r|l|}
\hline Journal: & Philosophical Magazine \& Philosophical Magazine Letters \\
\hline Manuscript ID: & TPHM-06-Oct-0421 \\
\hline Journal Selection: & Philosophical Magazine \\
\hline Author: & 18 -Oct-2006 \\
\hline Keywords: & liquid crystals, disclinations \\
\hline Complete List of Authors: & $\begin{array}{l}\text { Cermelli, Paolo; University of Torino, Mathematics } \\
\text { Di Scala, Antonio; Politecnico di Torino, Mathematics }\end{array}$ \\
\hline Keywords (user supplied): & interfaces in nematics, disclination cores, layers in smectics C \\
\hline $\begin{array}{l}\text { Note: The following files were submitted by the author for peer review, but cannot be converted } \\
\text { to PDF. You must view these files (e.g. movies) online. }\end{array}$ \\
\hline cermelli-discala-revised.TEX
\end{tabular}

\section{(s) ScholaroNE \\ Manuscript Central}


/ / PhilosophicaLMagazine/\& Philoşphical Magazine ketter\$age 2 of 27
20 21
22
$1 / 1 / 1$

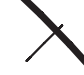
$1 /$
,
$x$
$1,-1$

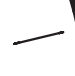 27 $28 \backslash$ $\backslash \backslash \backslash \backslash$

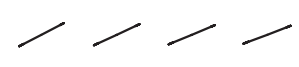




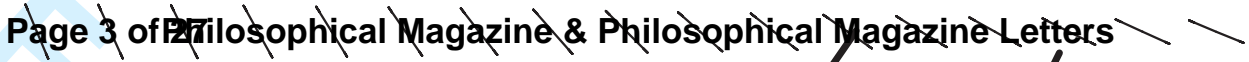

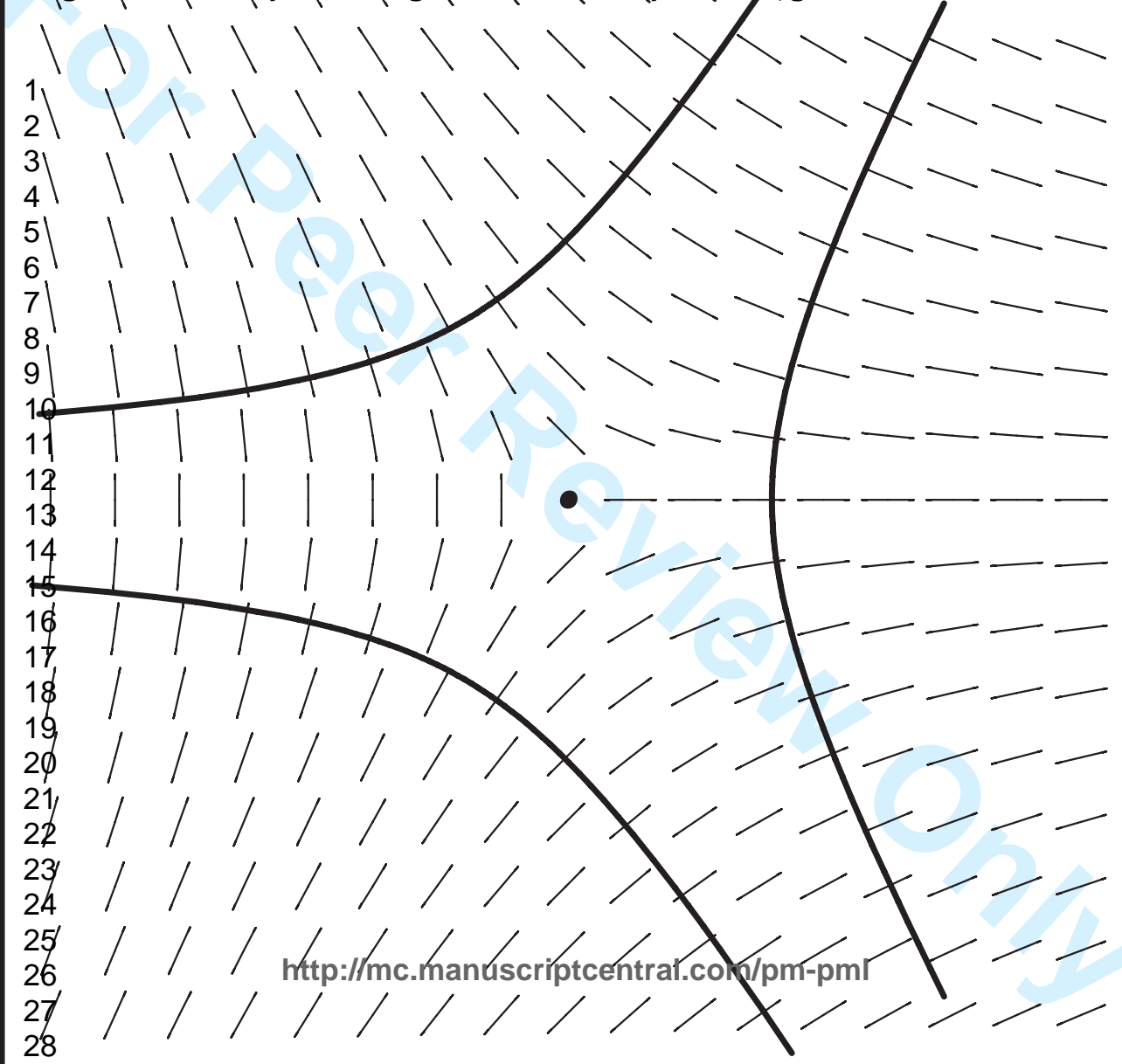


/ / Philøsophteal Magazine \& Philosophical Magazine Letter\$age 4 of 27

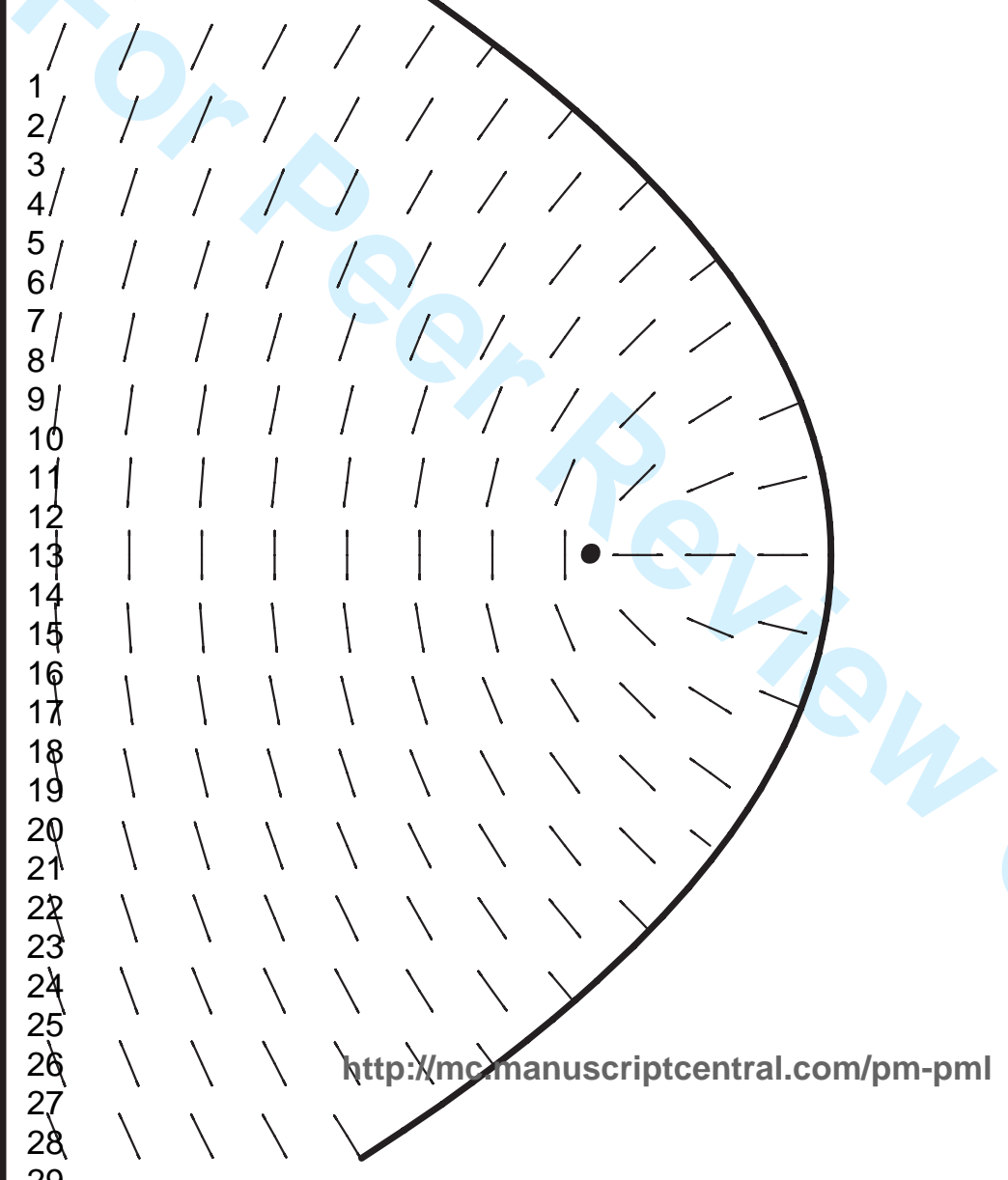


Page 5 of Errilosophical Magazine \& Philosophical Magazine_Letters

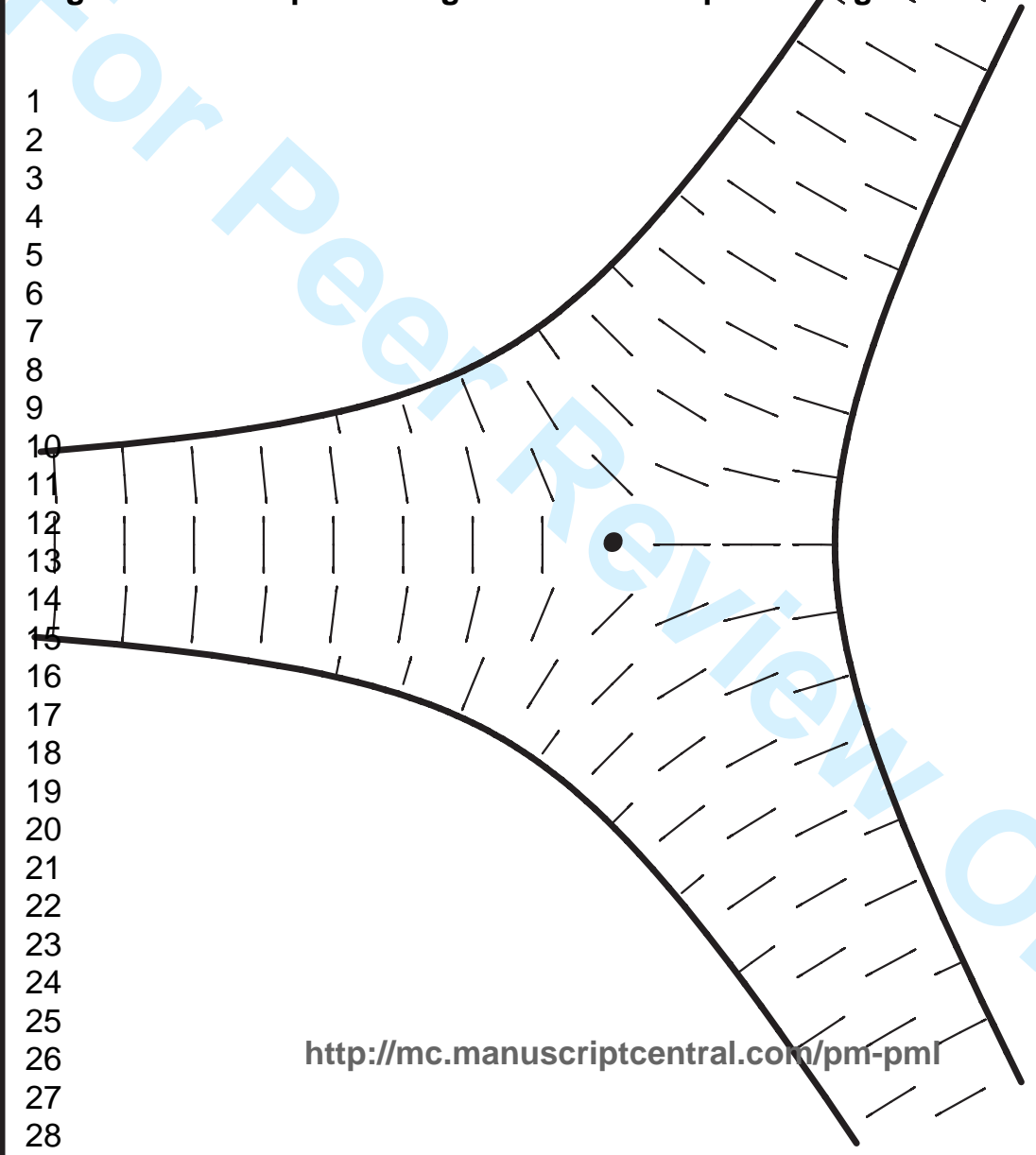




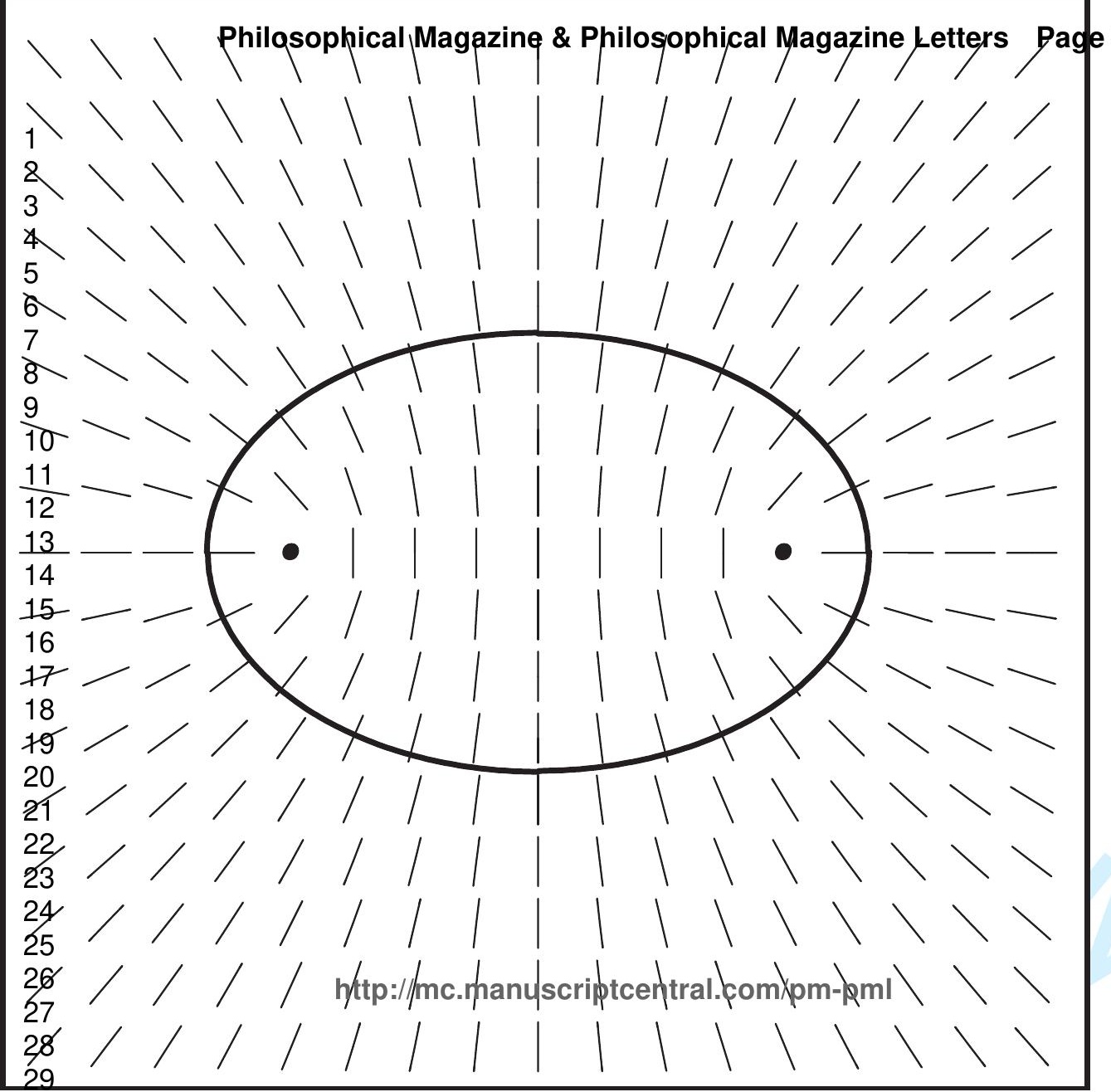


Page 7 offzrilosophical Magazine \& Philosophical Magazine Letters

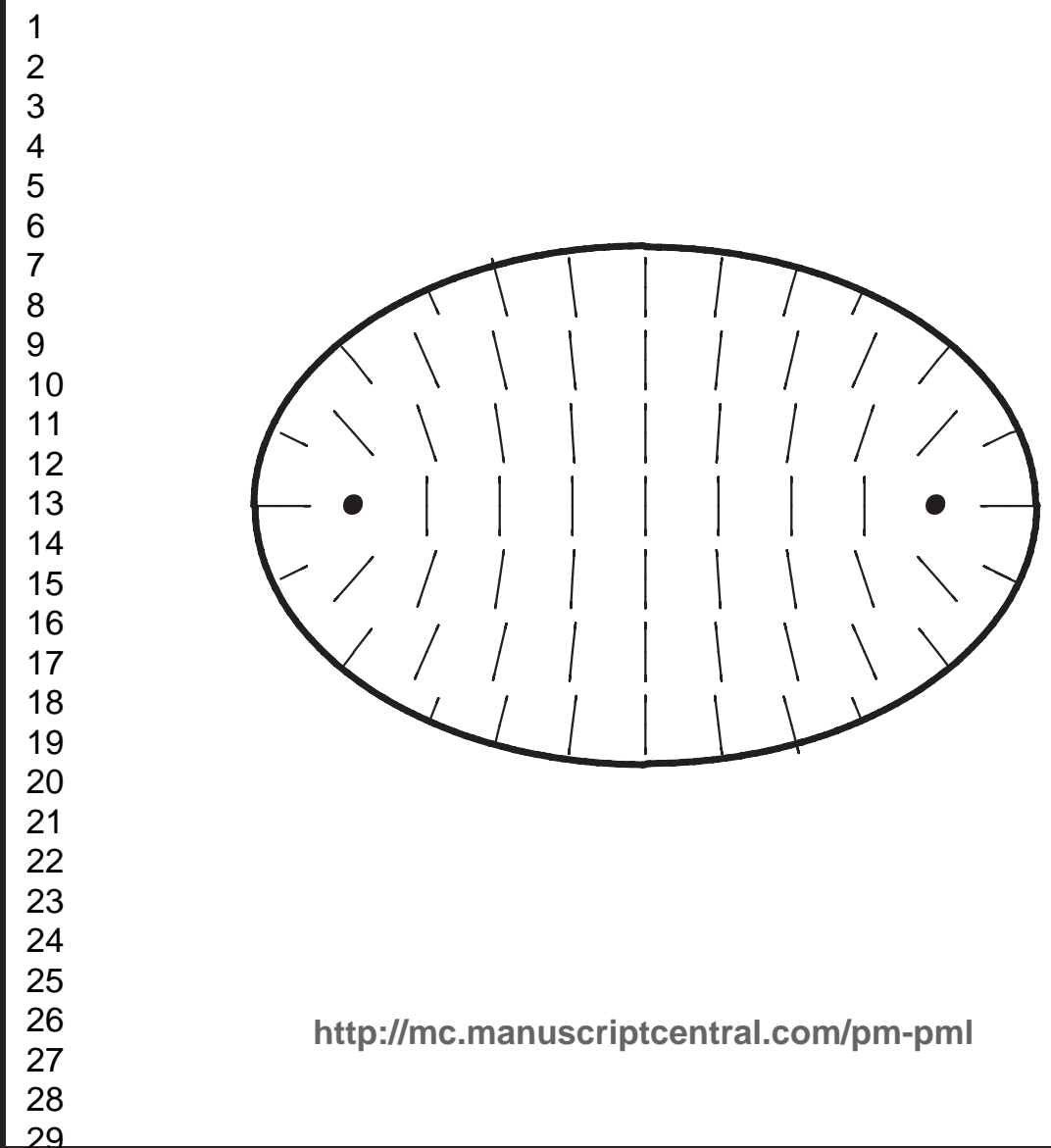


sophical Magazine \&_Philosophic Aladegaafnes Li

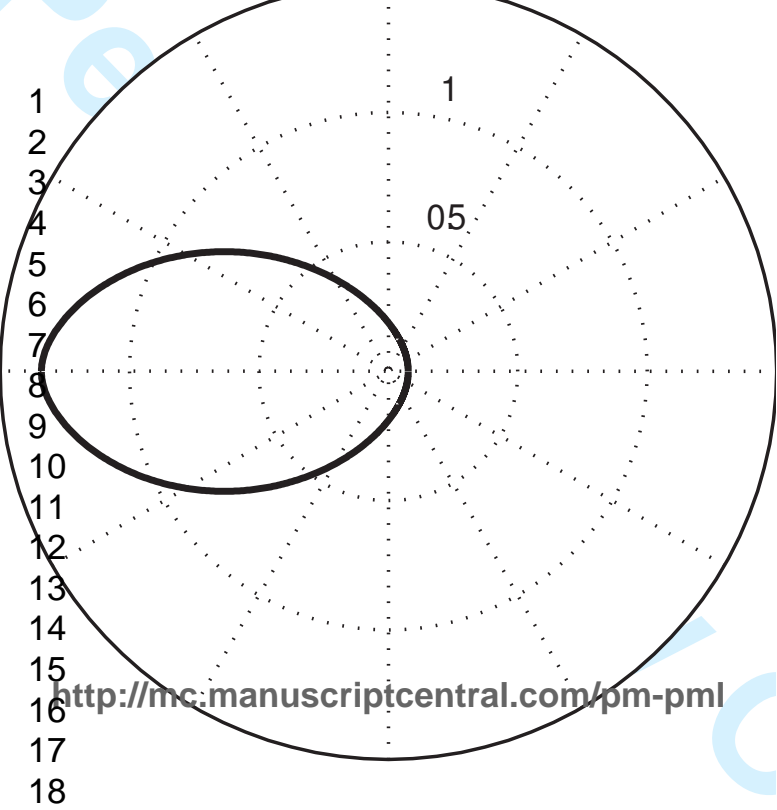


Page 9 of 2\$Philosophícal Magazzine/\& Philoşophical Magazine Ketters
$1 /$
$1 / 111$
I
$1<1$

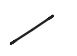
1
$1 / 1 / 1$
I
I

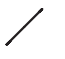
$3 /$
$1 /$
$1 /$
/
11
I
$\begin{array}{llll}5 & 1 & /\end{array}$
$1 / 1 /$
7
8
9
11
1
11
$1 /$
9
10
9
11
12
13
14
15
16
17
18
19
1
1
2
20
21
11
22
23
11
24
28
$26)$
27
$28 \backslash$
11
$\backslash$
$\backslash \backslash$ http://hnc.manuscriptcentral.com/smm-pml $\backslash>>$
$1 \backslash 1\rangle$
$\backslash$
$1 \backslash 1$
$\backslash \backslash \backslash$
$\backslash$
$+$
$\backslash \backslash 1$
$\backslash \backslash\rangle$
$\backslash>$
$\gamma>$
111111
$>$
$>>>>>>$ 11111111

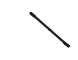
$\backslash$ 
sophical Magazine \& Philosophiectiglagaøin?

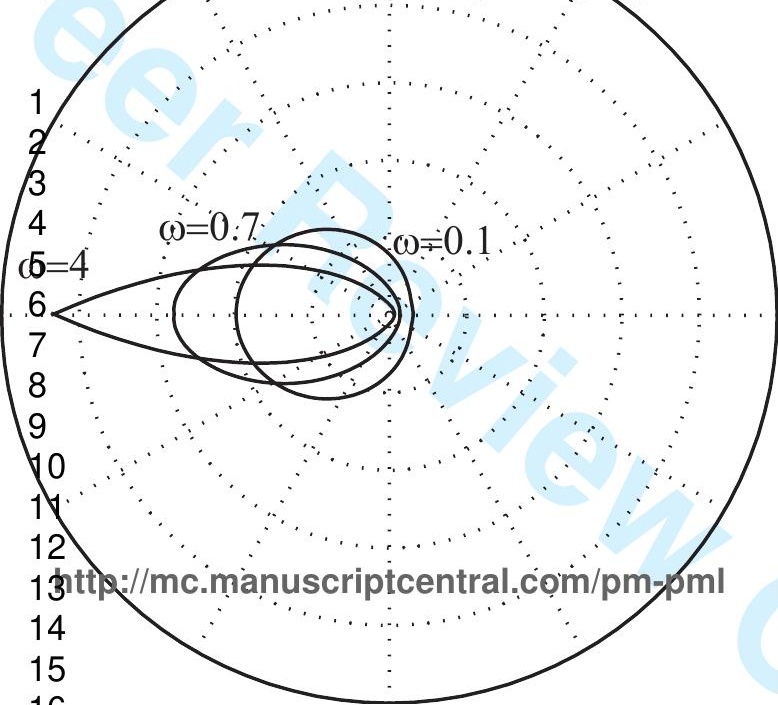


sopargieal Melgazine \&_Philosophical Magazine L

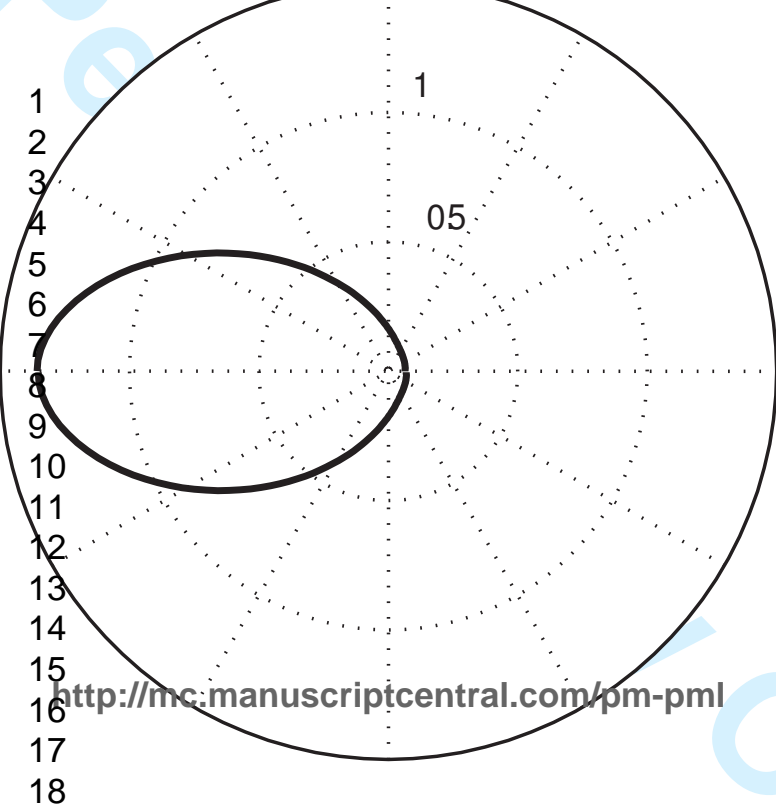


Phillosophical Magazine \& Philosophical MalgaginłZefters

$>$

$1 \backslash$

2\}

$3 \backslash$

5

$6-$

$7-$

8

9

10

11

12

13

14

15

16.

17

18

19

20

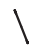

1
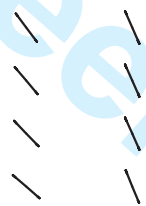

1

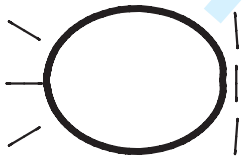

I

/

I

I

I

/

htt

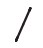

I

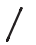

I
1
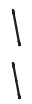

1
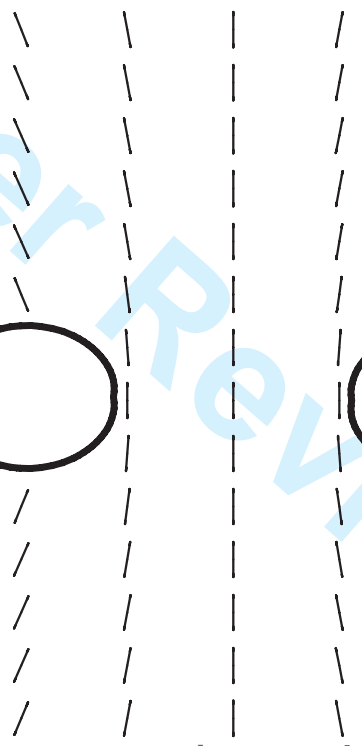

1

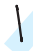

1

1

1

1

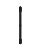

I

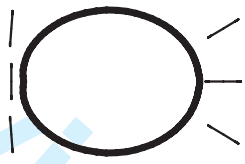

/
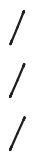

I

I
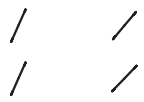

1

1
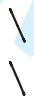

1

1
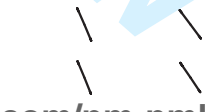

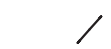


Philosophical Magazine,

\title{
Constant-angle surfaces in liquid crystals
}

\author{
Paolo Cermelli* \& Antonio J. Di Scala \\ Dipartimento di Matematica, Università di Torino, Via Carlo Alberto 10, 10123 Torino, Italy \\ Dipartimento di Matematica, Politecnico di Torino, Corso Duca degli Abruzzi 24, 10129 Torino, Italy \\ (Received 00 Month 200x; in final form 00 Month 200x)
}

\begin{abstract}
We discuss some properties of surfaces in $\mathbb{R}^{3}$ whose unit normal vector has constant angle with an assigned direction field. The constant angle condition can be rewritten as an Hamilton-Jacobi equation correlating the surface and the direction field. We focus on examples motivated by the physics of interfaces in liquid crystals and of layered fluids, and examine some properties of the constant-angle surfaces when the direction field is singular along a line (disclination) or at a point (hedgehog). We also show how our results may be used to study the shape of disclination cores.
\end{abstract}

\section{Introduction}

We study here surfaces in $\mathbb{R}^{3}$ whose unit normal vector forms a constant angle with an assigned direction field. The main idea is to show that the constant angle condition is equivalent to an Hamilton-Jacobi equation correlating the surface and the direction field. We discuss examples motivated by the physics of interfaces in liquid crystals and of layered fluids, and study the properties of constant-angle surfaces when the direction field is singular along a line or at a point. Precisely, we show how constant-angle surfaces may be used to describe interfaces occurring in special equilibrium configurations of nematic and smectic $\mathrm{C}$ liquid crystals, and to determine the shape of disclination cores in nematics (for an extensive study of boundaries of nematic drops or tactoids in nematics, in which the constant angle condition is relaxed, see Virga [1], [2], Prinsen and van der Schoot [3], [4], [5] and Calderer, Liu and Shen [6]).

The Hamilton-Jacobi approach to the determination of constant angle surfaces has been also applied extensively to the shape-from-shading problem (cf. e.g., Lions, Rouy and Tourin [7]), and specific numerical methods are now available for the computation of viscosity solutions of Hamilton-Jacobi equations (cf. e.g., Osher and Sethian [8] and Sethian [9]).

Precisely, a direction field on a domain $\Omega \subset \mathbb{R}^{3}$ can be locally described by a vector field $\boldsymbol{m}$ such that $|\boldsymbol{m}|=1$. However, since the vectors $\boldsymbol{m}$ and $\boldsymbol{- m}$ define the same direction, it is customary to assign a direction field through a map (which we continue to denote by the same symbol) $\boldsymbol{m}: \Omega \rightarrow \mathbb{P}^{2}(\mathbb{R}$ ), with $\mathbb{P}^{2}(\mathbb{R})$ the real projective plane, i.e., the set of all lines in $\mathbb{R}^{3}$ passing through the origin or, equivalently, the set of equivalence classes of unit vectors of the form $\{\boldsymbol{m},-\boldsymbol{m}\}$.

Consider a piecewise smooth surface $S \subset \Omega$ whose unit normal $\boldsymbol{n}$ has a constant angle $\alpha$ with $\boldsymbol{m}$ :

$$
|\boldsymbol{n} \cdot \boldsymbol{m}|=\cos \alpha,
$$

for fixed $\alpha \in\left[0, \frac{\pi}{2}\right]$. Viewing $S$ as a level set of a Lipschitz continuous function $f: \Omega \rightarrow \mathbb{R}$,

$$
S=\{\boldsymbol{x} \in \Omega / f(\boldsymbol{x})=\text { const. }\},
$$

and letting $\boldsymbol{n}=\nabla f /|\nabla f|,(1)$ is equivalent to the quadratic Hamilton-Jacobi equation

$$
\nabla f \cdot \boldsymbol{A}_{\alpha} \nabla f=0
$$

\footnotetext{
${ }^{*}$ Corresponding author. Email: paolo.cermelli@unito.it 
with $\boldsymbol{A}_{\alpha}=\boldsymbol{A}_{\alpha}(\boldsymbol{x})=\cos ^{2} \alpha \mathbf{1}-\boldsymbol{m}(\boldsymbol{x}) \otimes \boldsymbol{m}(\boldsymbol{x})$. Writing $H(\boldsymbol{x}, \boldsymbol{p})=\boldsymbol{p} \cdot \boldsymbol{A}_{\alpha}(\boldsymbol{x}) \boldsymbol{p}$ for the Hamiltonian, it is well known that local solutions of (2) may be obtained by the method of characteristics, which are the solutions of the canonical system (see Arnold [10])

$$
\dot{\boldsymbol{x}}=\frac{\partial H}{\partial \boldsymbol{p}}, \quad \dot{\boldsymbol{p}}=-\frac{\partial H}{\partial \boldsymbol{x}} .
$$

When $\alpha=0$ the method of characteristics fails, since the right-hand side of (3) vanishes identically for $H(\boldsymbol{x}, \boldsymbol{p})=0$. Indeed, when $\alpha=0, \boldsymbol{A}_{\alpha}$ is positive semidefinite, and the equation $H(\boldsymbol{x}, \boldsymbol{p})=0$ leads to an overdetermined system that must be studied by Frobenius theory. Thus, solutions of (1) for $\alpha=0$ exist if and only if $\boldsymbol{m} \cdot \operatorname{curl} \boldsymbol{m}=0$ in $\Omega$. In what follows we restrict to $\alpha \in(0, \pi / 2)$ when using the Hamilton-Jacobi approach, with the exception of Section 3.1 where the integrability condition is automatically satisfied and complex-variable methods can be applied.

In this work we study (2) for some special choices of the direction field $\boldsymbol{m}$. Namely, we characterize (i) cylindrical and conical surfaces arising when $\boldsymbol{m}$ is singular along a straight line and orthogonal to that line (line defects in nematics), and (ii) conical surfaces corresponding to a constant orientation field $\boldsymbol{m}=\boldsymbol{m}_{0}$ (layered structures in smectics C).

We now discuss briefly two physical examples which lead to equations of the form (1).

\section{$1.1 \quad$ Applications to nematics}

A nematic liquid crystal is an ordered fluid whose constituents are macromolecules which locally tend to align parallel to each other (cf., e.g., De Gennes and Prost [11]). A nematic liquid crystal can be described by a direction field $\boldsymbol{m}$ that measures the average local orientation of the macromolecules. In the simplest approximation, the field $\boldsymbol{m}$ at equilibrium is a stationary point of the (one-constant) Frank energy functional

$$
\int_{\Omega} \frac{\kappa}{2}|\nabla \boldsymbol{m}|^{2} d v
$$

with $\kappa>0$, whose Euler equation is the harmonic mapping equation (see Eells and Sampson [12] where (5) is introduced and studied in a general setting)

$$
\triangle \boldsymbol{m}+|\nabla \boldsymbol{m}|^{2} \boldsymbol{m}=0 .
$$

Often, the direction field describing the orientational order in a nematic fluid is not defined on the whole $\Omega$, the most common singularities being straight disclinations and hedgehogs.

Concisely, a (straight) disclination $\ell$ is a line in $\mathbb{R}^{3}$ such that $\boldsymbol{m}$ is not defined on $\ell$ but is smooth in $\Omega \backslash\{\ell\}$. Restricting to situations such that $\boldsymbol{m}$ is a plane vector field orthogonal to $\ell$, a disclination is characterized by its integer strength $k$, i.e., the degree of the mapping $\boldsymbol{m}: \gamma \rightarrow \mathbb{P}^{1}(\mathbb{R})$, with $\gamma$ a loop in $\Omega \backslash\{\ell\}$ encircling $\ell$ once. Roughly, $k$ is the net number of $\pi$-turns of $\boldsymbol{m}$ when $\gamma$ is traversed once (cf. Section 3).

A hedgehog is a point defect in $\mathbb{R}^{3}$ such that $\boldsymbol{m}$ is not defined at some point $\boldsymbol{x}_{0}$, but is smooth in $\Omega \backslash\left\{\boldsymbol{x}_{0}\right\}$. The simplest example of hedgehog, and the one we shall study here, is $\boldsymbol{m}(\boldsymbol{x})=\boldsymbol{x} /|\boldsymbol{x}|$ for $\boldsymbol{x} \neq \mathbf{0}$.

When singularities are present, it is usually assumed that, at equilibrium, $\boldsymbol{m}$ satisfies (5) away from the singularity.

Equations of the form (1) arise in the study of free surfaces of nematic liquid crystals and interfaces between nematic and isotropic phases. Indeed, denoting by $\boldsymbol{n}: \partial \Omega \rightarrow S^{2}$ the outward unit normal to $\partial \Omega$, which we view as the interface between the nematic and isotropic phase, it is often assumed that the strong anchoring boundary condition holds at $\partial \Omega$

$$
|\boldsymbol{n} \cdot \boldsymbol{m}|=\cos \alpha,
$$


with $\alpha \in(0, \pi / 2)$, which is an equation of the form (1).

In Sections 2 and 3 we restrict to two special cases: first, assuming that $\boldsymbol{m}$ is a constant solution of (5), we show that surfaces satisfying (1) are flat and ruled, and can be explicitly described. Second, assuming that $\boldsymbol{m}$ has a line singularity with arbitrary integer strength, we study cylindrical surfaces which satisfy (1), and discuss how their topology depends on the strength.

However, since in general surfaces solutions of (1) are not bounded, they cannot be used to describe the boundaries of nematic drops (tactoids). Actually, (1) must be relaxed by requiring that the director field at the surface $\partial \Omega$ satisfies weak anchoring conditions, i.e., that $\boldsymbol{m}$ and $\partial \Omega$ minimize the total energy, given by the sum of the Frank energy (4) and a surface energy term of the form (cf. Rapini and Papoular [13])

$$
\int_{\partial \Omega}\left(\psi_{0}+\psi(\boldsymbol{n} \cdot \boldsymbol{m})\right) d a
$$

where $\psi_{0}>0$ is a fixed constant and $\psi$ is the anchoring energy, which is assumed to have absolute minima at $\boldsymbol{n} \cdot \boldsymbol{m}= \pm \cos \alpha$.

The energy-minimizing approach to determine the optimal shape of tactoids has been extensively developed by Virga [1], [2] and, more recently, by Prinsen and van der Schoot [3], [4], [5] and Calderer, Liu and Shen $[6]$.

In this paper we do not discuss the optimal shape of nematic drops, but we make use of the energyminimizing approach to study the shape of the core of a straight disclination. In an oversimplified model, the core of a defect may be viewed as an isotropic inclusion in a nematic environment: in this case, since the inclusion has a volume which is negligible with respect to the environment, scaling arguments show that we may assume that the nematic field is a given singular solution of (5) outside the defect core, whose boundary is a minimizer of the surface energy (6) (cf. the related work by Rosso and Brunelli [14], who determine the optimal shape of a disclination core neglecting surface energy).

\section{$1.2 \quad$ Applications to smectics $C$}

Smectic C liquid crystals are ordered fluids characterized by a layered structure (cf. De Gennes and Prost [11]). When density fluctuations are neglected, the description of equilibrium configurations of smectics requires, in addition to the orientation field $\boldsymbol{m}$, a scalar order parameter $f: \Omega \rightarrow \mathbb{R}$ whose level sets define the layers. In smectics $\mathrm{C}$, the director field $\boldsymbol{m}$ is constrained to a constant angle $\alpha$ with the layer normal $\boldsymbol{n}=\nabla f /|\nabla f|$, i.e.,

$$
|\boldsymbol{m} \cdot \boldsymbol{n}|=\cos \alpha,
$$

with $\alpha \in(0, \pi / 2)$.

The energy of a smectic $\mathrm{C}$ liquid crystal may be written as the sum of a nematic energy term, which in the simplest approximation is the Frank energy (4), and a smectic term of the form (cf. [11], [15])

$$
\int_{\Omega}\left(A|\nabla f-q \boldsymbol{m}|^{2}+B|\boldsymbol{m} \cdot \nabla f-q|^{2}\right) d v
$$

where $A, B, q$ are positive constants.

An energetically-based approach to the determination of layered structures in smectics, which allows the director field to vary, is developed by Kidney, McKay and Stewart [16], McKay and Leslie [17], and McKay [18]. We restrict here to special configurations such that:

- the orientation field $\boldsymbol{m}$ is assigned;

- the smectic order parameter is an absolute minimizer of the smectic energy density (7) subject to the constraint (1).

Letting $\boldsymbol{P}=\mathbf{1}-\boldsymbol{m} \otimes \boldsymbol{m}$, then $|\nabla f|^{2}=|\boldsymbol{P} \nabla f|^{2}+(\boldsymbol{m} \cdot \nabla f)^{2}$ and, granted the constraint (1), then also 
$|\boldsymbol{P} \nabla f|^{2}=\tan ^{2} \alpha(\boldsymbol{m} \cdot \nabla f)^{2}$, so that the integrand of (7) may be rewritten as

$$
\left(A+B+A \tan ^{2} \alpha\right)(\boldsymbol{m} \cdot \nabla f)^{2}-2 q(A+B)(\boldsymbol{m} \cdot \nabla f)+q^{2}(A+B),
$$

and minimization with respect to $x=(\boldsymbol{m} \cdot \nabla f)$ yields the system of PDEs

$$
\left\{\begin{array}{l}
|\boldsymbol{P} \nabla f|=\gamma \tan \alpha \\
\boldsymbol{m} \cdot \nabla f=\gamma
\end{array}\right.
$$

with $\gamma=\frac{(A+B) q}{(A+B)+A \tan ^{2} \alpha}$. We show below that, contrary to (2), (8) has no solution for a large class of orientation fields, and discuss two cases when the solution does exist, i.e., for a uniform orientation field, and a line disclination with strength 2 .

\section{Constant-angle conical surfaces in a uniform orientation field}

For a uniform orientation field $\boldsymbol{m}=\boldsymbol{m}_{0}$ on $\mathbb{R}^{3}$,(1) reduces to

$$
\left|\boldsymbol{n} \cdot \boldsymbol{m}_{0}\right|=\cos \alpha
$$

and we assume that $\alpha \in(0, \pi / 2)$.

Let $S$ be a solution of (9): notice first that smooth portions of $S^{\prime} \subset S$ are flat, since the Gaussian curvature $K$ identically vanishes on these portions. By consequence, $S^{\prime}$ is also ruled.

To see this, let $S^{\prime} \subset S$ be smooth, and notice that (9) implies that the image of $S^{\prime}$ by the Gauss map $\boldsymbol{n}: S^{\prime} \rightarrow S^{2}$ is a circle. By consequence, the Gaussian curvature vanishes, since

$$
K=\lim _{\operatorname{area}\left(S^{\prime}\right) \rightarrow 0} \frac{\operatorname{area}\left(\boldsymbol{n}\left(S^{\prime}\right)\right)}{\operatorname{area}\left(S^{\prime}\right)}=0
$$

and $S^{\prime}$ is flat.

To solve (9), choose Cartesian coordinates $(x, y, z)$, with associated basis $(\boldsymbol{i}, \boldsymbol{j}, \boldsymbol{k})$ such that $\boldsymbol{k}=\boldsymbol{m}_{0}$. Assume that $S$ is smooth and is locally the graph of a function $g:=D \subset \mathbb{R}^{2} \rightarrow \mathbb{R}$, i.e.,

$$
S=\{(x, y, z) / z=g(x, y)\}
$$

Since $\boldsymbol{n}=(-\nabla g+\boldsymbol{k}) / \sqrt{1+|\nabla g|^{2}}$, with $\nabla g=\left(\partial_{x} g\right) \boldsymbol{i}+\left(\partial_{y} g\right) \boldsymbol{j},(9)$ reduces to the eikonal equation

$$
|\nabla g|=\tan \alpha
$$

Let $C_{0}=S \cap\{z=0\}$ be the trace of $S$ on the $(x, y)$-plane. Then $S$ is a piece of a conical surface generated by $C_{0}$. To see this, let $\boldsymbol{x}_{0}(s)$ be a parametrization of $C_{0}$ with arc parameter $s$, and let $\boldsymbol{\tau}=\boldsymbol{\tau}(s)$ and $\boldsymbol{\nu}=\boldsymbol{\nu}(s)$ be the unit tangent and normal vectors to $C_{0}$. Writing $\boldsymbol{x}=x \boldsymbol{i}+y \boldsymbol{j}$, let also $t=t(\boldsymbol{x}):=\operatorname{dist}\left(\boldsymbol{x}, C_{0}\right)$ be the distance of a point $\boldsymbol{x}$ from $C_{0}$ (which is well defined in a neighborhood of the curve if $C_{0}$ is smooth), and notice that the solution of (10) with boundary condition $g(x, y)=0$ on $C_{0}$ is

$$
g(x, y)=t(x, y) \tan \alpha
$$

In fact, consider the local change of coordinates $((s, t)$ are known as Fermi coordinates)

$$
\boldsymbol{x}(s, t)=\boldsymbol{x}_{0}(s)+t \boldsymbol{\nu}(s),
$$


and let

$$
\hat{g}(s, t):=g(\boldsymbol{x}(s, t))
$$

Since $\nabla g \cdot \boldsymbol{\nu}=\partial_{t} \hat{g}$, and $\nabla g \cdot \boldsymbol{\tau}=\partial_{s} \hat{g} /(1-c t)$, with $c$ the curvature of $C_{0}$, it follows, by taking $\hat{g}(s, t)=t \tan \alpha$, that $\nabla g=\tan \alpha \boldsymbol{\nu}$, so that (10) is satisfied.

As is well known, (11) is a local solution of (10), since the distance is in general a multi-valued function. However, the method of characteristics actually yields a surface $S$ in $\mathbb{R}^{3}$ which is a global solution of the original equation (9), given by the global parametrization

$$
\boldsymbol{x}_{0}(s)+t \boldsymbol{\nu}(s)+(t \tan \alpha) \boldsymbol{m}_{0},
$$

in terms of the Fermi coordinates $(s, t)$ as $t$ varies in $\mathbb{R}$. The surface $S$ parametrized by (12) may have self-intersections, for instance at points $(s, t)$ corresponding to the caustics of (10). In Figure 1 a portion of a conical surface with $C_{0}$ an ellipse is shown: notice the sharp edge where the generatrices meet corresponding to the caustics of the eikonal inside the ellipse.
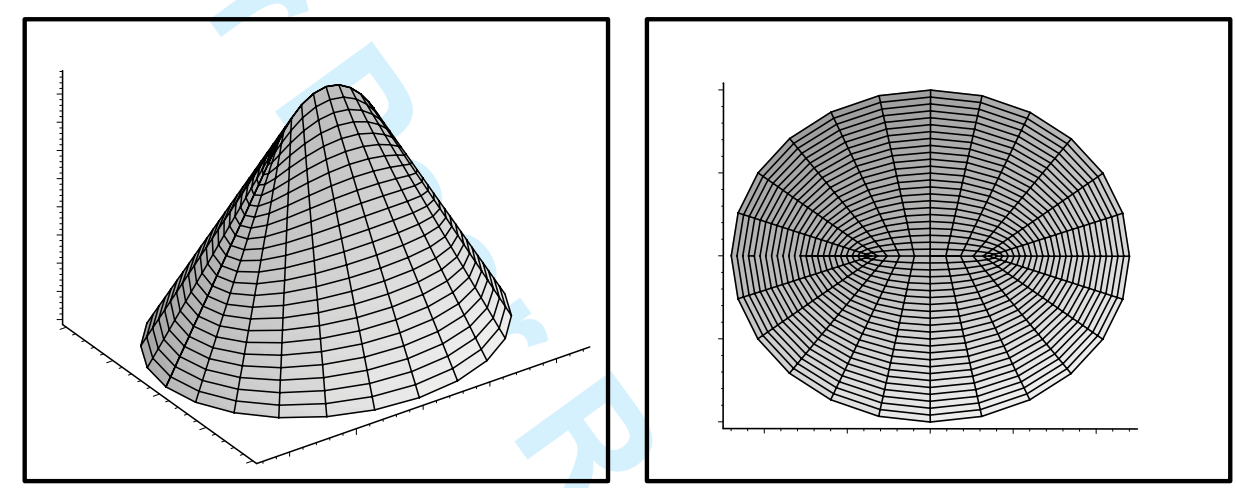

Figure 1. Side and top views of a portion of a conical constant-angle surface with vertical uniform orientation field $\boldsymbol{m}=\boldsymbol{m}_{0}$. The plane curve generating the cone is an ellipse.

\section{Cylindrical surfaces in the presence of a straight disclination}

Let $\Omega=D \times \mathbb{R}$ be a cylindrical domain, with $D \subset \mathbb{R}^{2}$, and consider an orientation field $\boldsymbol{m}: \Omega \rightarrow \mathbb{P}^{2}(\mathbb{R})$. Assume that $\boldsymbol{m}$ is planar and independent of the vertical coordinate, so that

$$
\boldsymbol{m}=\boldsymbol{m}(\boldsymbol{x}), \quad \boldsymbol{m} \cdot \boldsymbol{k}=0,
$$

with $\boldsymbol{x}=x \boldsymbol{i}+y \boldsymbol{j} \in D$, and $(\boldsymbol{i}, \boldsymbol{j}, \boldsymbol{k})$ an orthonormal basis with $\boldsymbol{k}$ orthogonal to the plane containing $D$. Under the above assumptions the problem is 2-dimensional and we may view $\boldsymbol{m}: D \rightarrow \mathbb{P}^{1}(\mathbb{R})$.

Since $\boldsymbol{m}$ is planar, we may write

$$
\boldsymbol{m}(\boldsymbol{x})=\cos \varphi(\boldsymbol{x}) \boldsymbol{i}+\sin \varphi(\boldsymbol{x}) \boldsymbol{j}
$$

Notice that, for nematic orientation fields, $\boldsymbol{m}$ satisfies (5) if and only if $\varphi$ is harmonic:

$$
\Delta \varphi=0
$$

with $\Delta$ the laplacian in $\mathbb{R}^{2}$. 


\subsection{Surfaces orthogonal to $m: \alpha=0$}

We first look for surfaces $S \subset \Omega$ such that

$$
|\boldsymbol{n} \cdot \boldsymbol{m}|=1
$$

with $\boldsymbol{n}$ a choice of unit normal for $S$. Since $\boldsymbol{m} \cdot \operatorname{curl} \boldsymbol{m}=0$, Frobenius theorem is trivially satisfied and local solutions to (15) exist. Moreover, the same argument of Section 2 shows that the corresponding surfaces are flat and ruled, actually cylinders $S=C \times \mathbb{R}$ with vertical axis, with $C \subset D$ a curve in the plane orthogonal to $\boldsymbol{k}$.

Noting that the plane curve $C$ has unit normal $\boldsymbol{n}$, it follows that $C$ is still defined by (15), which we henceforth view as an equation in the unknown curve $C$.

Equation (15) defines the family of (orthogonal) integral curves of the direction field $\boldsymbol{m}$. Describing $C$ as a level set of a function $f: D \subset \mathbb{R}^{2} \rightarrow \mathbb{R}$ :

$$
C=\{\boldsymbol{x} \in D: f(\boldsymbol{x})=c\},
$$

with $c \in \mathbb{R},(15)$ is equivalent to

$$
\nabla f \times \boldsymbol{m}=0 .
$$

Assume first that $D$ is simply connected and $\boldsymbol{m}$ is a smooth solution of (5) in $\Omega$, so that no defect is present and $\varphi$ is harmonic in $D$. Denote by $w$ the harmonic conjugate of $\varphi$, solution of the Cauchy-Riemann equations

$$
\frac{\partial \varphi}{\partial x}+\frac{\partial w}{\partial y}=0, \quad \frac{\partial \varphi}{\partial y}-\frac{\partial w}{\partial x}=0
$$

and define

$$
\boldsymbol{u}=e^{-w} \boldsymbol{m}=e^{-w}(\cos \varphi \boldsymbol{i}+\sin \varphi \boldsymbol{j}),
$$

so that $\boldsymbol{u}$ is a non-zero vector field on $D$. The function $e^{-w}$ is an integrating factor for $\boldsymbol{m}$, and it is easy to see that Div $\boldsymbol{u}=0$ and $\operatorname{Curl} \boldsymbol{u}=0$, where Curl $\boldsymbol{u}=u_{x}^{2}-u_{y}^{1}$ for $\boldsymbol{u}=u^{1}(x, y) \boldsymbol{i}+u^{2}(x, y) \boldsymbol{j}$. By consequence, $\Delta \boldsymbol{u}=0$ in $D$. In particular, there exists $f: D \rightarrow \mathbb{R}$ such that

$$
\nabla f=\boldsymbol{u}
$$

so that $\boldsymbol{n}=\nabla f /|\nabla f|=\boldsymbol{m}$ and $\{f(\boldsymbol{x})=c\}$ is an orthogonal integral curve of the direction field $\boldsymbol{m}$, i.e., is a solution of (15).

Equivalently, identify $\mathbb{R}^{2}$ to $\mathbb{C}$ and, for $z=x+i y$, define

$$
m(z)=e^{-i \varphi(z)} \quad \text { and } \quad u(z)=e^{-(w(z)+i \varphi(z))},
$$

where $w(z)$ and $\varphi(z)$ are $w(x, y)$ and $\varphi(x, y)$ expressed in terms of the complex variable $z$. By construction $u$ is holomorphic in $D$.

Consider now a holomorphic function in $D$

$$
F=f+i g,
$$

with $f$ and $g$ real valued. Since $F$ is holomorphic,

$$
F^{\prime}=2 \frac{\partial f}{\partial z}=\frac{\partial f}{\partial x}-i \frac{\partial f}{\partial y} .
$$


Comparing (18) and (19) we conclude that $f$, as a function on $D \subset \mathbb{R}^{2}$, satisfies (15) and (16) if and only if $F$ as defined by (20) satisfies

$$
F^{\prime}(z)=\lambda(z) u(z)
$$

for some real function $\lambda(z)$ on $D$. However, since $F^{\prime}$ and $u$ are holomorphic, $\lambda$ is a real constant, so that (15) and (16) reduce (modulo a multiplicative real constant) to

$$
F^{\prime}(z)=u(z)
$$

Therefore, the plane curves solutions of (15) are the level sets of the real part $f$ of any primitive $F$ of $u$, i.e.,

$$
C=\{z \in \mathbb{C}: \operatorname{Re}(F(z))=c\}
$$

Going back to three dimensions, assume that the director field $\boldsymbol{m}$ is singular along a line $\ell$ parallel to the axis of the cylinder $\Omega$ : precisely, assume that $\ell$ is a straight disclination of strength $k$. Assuming that $\ell$ intersects $D$ at $\boldsymbol{x}=\mathbf{0}$, we may henceforth restrict to the plane direction field $\boldsymbol{m}: D \backslash\{\mathbf{0}\} \rightarrow \mathbb{P}^{1}(\mathbb{R})$, which is singular at $\mathbf{0}$. Recall now that the strength $k \in \mathbb{Z}$ of the disclination at $\mathbf{0}$ is defined as the net number of $\pi$-rotations of $\boldsymbol{m}$ along any simple closed loop encircling $\mathbf{0}$, given by the relation

$$
\frac{k}{2}=\frac{1}{2 \pi} \int_{\gamma}\left(-m^{2} \nabla m^{1}+m^{1} \nabla m^{2}\right) \cdot d \boldsymbol{x}=\frac{1}{2 \pi} \int_{\gamma} d \varphi
$$

Fix now $k \in \mathbb{Z}$ : any solution of (14) with given strength $k$ may be written in the form

$$
\varphi(\varrho, \vartheta)=\frac{k}{2} \vartheta+g(\varrho, \vartheta)
$$

with $g: D \backslash\{\mathbf{0}\} \rightarrow \mathbb{R}$ harmonic (and single-valued) and $(\varrho, \vartheta)$ polar coordinates centered at $\{\mathbf{0}\}$. Therefore, by means of a conformal transformation ${ }^{1}$, we may restrict to director fields that have the form

$$
\boldsymbol{m}=\cos \left(\frac{k \vartheta}{2}\right) \boldsymbol{i}+\sin \left(\frac{k \vartheta}{2}\right) \boldsymbol{j}
$$

Switching to complex-variables, and letting $\varphi=k \vartheta / 2$ in (19) we obtain

$$
u(z)=z^{-\frac{k}{2}}
$$

Now recall that the orthogonal integral curves of $\boldsymbol{m}$ are given by (22), where $F$ is a primitive of $u$,

$$
F^{\prime}(z)=z^{-\frac{k}{2}}
$$

which yields

$$
F(z)= \begin{cases}\frac{2}{2-k} z^{\frac{2-k}{2}}, & k \neq 2, \\ \log z, & k=2 .\end{cases}
$$

\footnotetext{
${ }^{1}$ In complex coordinates the conformal (holomorphic) transformation is $z \mapsto z \exp \left(\frac{2}{k}\left(w_{g}+i g\right)\right)$, with $w_{g}$ the harmonic conjugate of $g$.
} 
so that the curve $C$ has the form $f(z)=c$, with

$$
f(z)= \begin{cases}\operatorname{Re}\left(z^{\frac{2-k}{2}}\right)=\frac{1}{2}\left(z^{\frac{2-k}{2}}+\bar{z}^{\frac{2-k}{2}}\right), & k \neq 2 \\ \log |z|, & k=2 .\end{cases}
$$

To avoid discussing the sign of $c$, consider the curves $C^{\prime}$ defined by $|f(z)|=c>0$. The topology of $C^{\prime}$ depends on $k$ as follows:

- For $k \leq 1, k \neq 0$, the curve $|f(z)|=c$ is not bounded, does not contain the defect $z=0$ and has $2-k$ connected components with $2-k$ asymptotic directions (cf. Figure 2). In polar coordinates $C$ has the explicit representation

$$
\varrho=\frac{c}{\left|\cos \left(\frac{2-k}{2} \vartheta\right)\right|^{\frac{2}{2-k}}} .
$$

- For $k=2$, the field $\boldsymbol{m}$ is radial and the curves $|f(z)|=c$ are circles centered at 0 .

- For $k \geq 3$ the curve $C^{\prime}$ contains the defect at $z=0$. $C^{\prime}$ is bounded, and has a clover-like shape with $k-2$ leafs. In polar coordinates $C^{\prime}$ has the explicit representation

$$
\varrho=c\left|\cos \left(\frac{k-2}{2} \vartheta\right)\right|^{\frac{2}{k-2}} .
$$
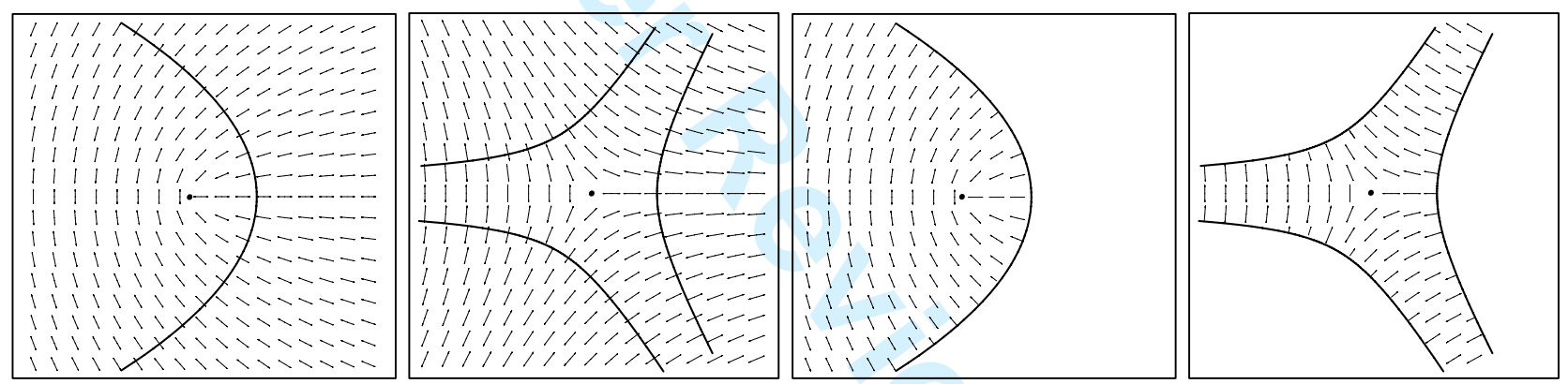

Figure 2. From left to right: (i) a section of a cylindrical constant-angle surface for $\alpha=0$, with a disclination of strength $k=1$ located along the axis of the cylinder (directed out of the page); (ii) same as before with $k=-1$;

(iii) a section of a cylindrical domain $K$ with boundary a constant angle surface: a bump for $k=1$ and (iv) a meniscus for $k=-1$.

\section{$3.2 \quad$ Surfaces with $\alpha$ arbitrary}

The above results may readily be extended to surfaces $S \subset \mathbb{R}^{3}$ such that

$$
|\boldsymbol{m} \cdot \boldsymbol{n}|=\cos \alpha,
$$

with $\alpha \in(0, \pi / 2)$. Again, solutions of (28) are cylinders $S=C \times \mathbb{R}$ with vertical axis, with $C \subset D$ a plane curve solution of $(28)$ in $D$.

Since (28) is equivalent to $|\overline{\boldsymbol{m}} \cdot \boldsymbol{n}|=1$, where $\overline{\boldsymbol{m}}=\cos (\varphi \pm \alpha) \boldsymbol{i}+\sin (\varphi \pm \alpha) \boldsymbol{j}$, all considerations in Section 3.1 hold, with

$$
u(z)=e^{-(w(z)+i(\varphi(z) \pm \alpha))} .
$$


Consider now, as in Section 3.1, a straight disclination with strength $k$ parallel to the axis of the cylinder, and the corresponding singular plane direction field $\boldsymbol{m}$ as in (24). In this case, (25) takes the form

$$
u(z)=e^{ \pm i \alpha} z^{-\frac{k}{2}}
$$

so that

$$
F(z)= \begin{cases}\frac{2}{2-k} e^{ \pm i \alpha} z^{\frac{2-k}{2}}, & k \neq 2 \\ e^{ \pm i \alpha} \log z, & k=2\end{cases}
$$

and it follows that the curve $C$ has the form $f(z)=c$, with

$$
f(z)= \begin{cases}\varrho^{\frac{2-k}{2}} \cos \left(\frac{2-k}{2} \theta \pm \alpha\right), & k \neq 2 \\ \log \varrho \pm \theta \tan \alpha, & k=2 .\end{cases}
$$

The curves $C^{\prime}$ defined by $|f(z)|=c>0$, are now such that

- For $k \neq 2$, the curve $C^{\prime}$ is a rigid rotation (of angle $\frac{2}{2-k} \alpha$ ) of the curves defined by (26) and (27).

- For $k=2$,the curves $|f(z)|=c$ are logarithmic spirals of the form

$$
\varrho=c e^{ \pm \theta \tan \alpha} .
$$

\subsection{Disclination pairs}

The above argument may be extended to any number $N$ of parallel dislocations with strength $k_{i}$, intersecting the horizontal plane at $\boldsymbol{z}_{i}, i=1, \ldots, N$ (complex representation $z_{i}$ ). Modulo a conformal transformation, at equilibrium the nematic angle may be written in the form

$$
\varphi=\varphi_{0}+\frac{1}{2} \sum_{i=1}^{N} k_{i} \vartheta_{i}
$$

with $\vartheta_{i}(\boldsymbol{x})=\arctan \frac{y-y_{i}}{x-x_{i}}$ a polar angle centered at $\boldsymbol{z}_{i}$ and $\varphi_{0}$ a constant angle. Proceeding as in the above section, we find in the complex representation

$$
u(z)=e^{-i \varphi_{0}} \prod_{i=1}^{N}\left(z-z_{i}\right)^{-\frac{k_{i}}{2}} .
$$

To be specific, consider two parallel disclinations of strength $k_{1}=k_{2}=1$, located at $\boldsymbol{z}_{1}=0$ and $\boldsymbol{z}_{2}=-a \boldsymbol{i}$, with $a>0$ (complex representation $z_{1}=0$ and $z_{2}=-a$ ), and assume that the nematic angle is given by

$$
\varphi=\frac{1}{2} \vartheta+\frac{1}{2} \bar{\vartheta}
$$

where $\bar{\vartheta}(\boldsymbol{x})=\arctan \frac{y}{x+a}$. Then

$$
u(z)=z^{-\frac{1}{2}}(z+a)^{-\frac{1}{2}}
$$

and a primitive $F$ of $u$ is easily calculated:

$$
F(z)=\log \left(z+\frac{a}{2}+\sqrt{z(z+a)}\right) .
$$




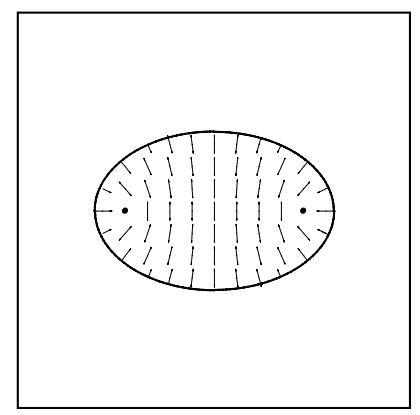

Figure 3. From left to right: (i) a section of cylindrical orthogonal surface surrounding a pair of parallel disclinations with strength $1 / 2$ and mutual separation $a=2$; (ii) a section of a capillary tube with shape a constant-angle surface: for orthogonal (homeotropic) anchoring at the walls an equilibrium configuration has two disclinations of strength $k=1$.

It follows that the orthogonal curves of the direction field with angle (29) are given by

$$
\left|z+\frac{a}{2}+\sqrt{z(z+a)}\right|=c,
$$

with $c$ a real positive constant. The explicit form of this curve in polar coordinates follows from a straightforward calculation, and will be omitted here. Figure 3 shows the plot of one such curve surrounding a pair of disclinations with separation $a=2$.

\section{Disclination cores in nematics}

Consider a singular orientation field $\boldsymbol{m}$ as in (24). Domains whose boundaries are constant-angle surfaces correspond to natural equilibrium interfaces between nematic and isotropic phases, since the orientation field inside the domain is by construction a solution of (5), and strong anchoring conditions are automatically satisfied at the interface. Specifically, consider a cylindrical domain $K=D^{\prime} \times \mathbb{R} \subset D \times \mathbb{R}$, with $C^{\prime}=\partial D^{\prime} \subset C$ a portion of a constant-angle surface. From the discussion in Section 3.1 it follows that

- For $k \leq 1, K$ is not bounded. This class of domains corresponds to curved bounding surfaces of domains containing a disclination $(k=1)$, or to menisci connecting films containing a disclination $(k=-1)$ (Figure 2). When $k=0$ we obtain a film in which the orientation field is aligned.

- For $k=2, K$ is either a circular cylindrical filament centered at the disclination, or its complement, which corresponds to a cylindrical isotropic core about a straight disclination.

- For a pair of disclinations of strength $k=1$, the domain $K$ is a filament with elongated section containing the disclination lines. This configuration may be realized for instance in a capillary tube with elongated section and orthogonal anchoring at the walls (Figure 3).

A second issue that can be discussed in this contest is the equilibrium shape of disclination cores. As mentioned above, in a crude approximation we may view the core as an isotropic inclusion in a nematic environment. For very small sectional areas of the core, the nematic field can be assumed to be a fixed equilibrium solution of (5) outside the core, and the shape of the core itself is a minimizer of the interfacial energy (6).

Precisely, assume that the nematic angle $\varphi$ is assigned, a fixed solution of (14) of the form (24). Let $D^{\prime}$ be the planar section of the disclination core, write $\boldsymbol{t}$ for a choice of unit tangent to $\partial D^{\prime}$, and consider the surface energy functional (per unit height of the cylinder)

$$
\int_{\partial D^{\prime}}\left(\psi_{0}+\omega(\boldsymbol{m} \cdot \boldsymbol{t})^{2}\right) d s
$$


with $\omega, \psi_{0}>0$ and $s$ the arc-parameter on the curve $\partial D^{\prime}$. Clearly, orthogonal anchoring is preferred at the core-boundary.

Consider the following problem: find the plane region $D^{\prime}$, containing the origin, that minimizes (32) under the constraint that area $\left(D^{\prime}\right)=1$. Noting that $\boldsymbol{m}=\nabla f /|\nabla f|,(32)$ may be written as

$$
\int_{\partial D^{\prime}}\left(\psi_{0}+\frac{\omega}{|\nabla f|^{2}}\left(\frac{\partial f}{\partial s}\right)^{2}\right) d s
$$

We only discuss the cases $k= \pm 1$. In polar coordinates centered at the origin (the trace of the disclination), let $\varrho=R(\vartheta)$ be the parametric representation of the surface $f=$ const., i.e., $\partial D^{\prime}$, and notice that we may restrict attention to curves that are symmetric with respect to the $x$-axis. Then the minimization of (32) is equivalent to the minimization of

$$
\int_{0}^{\pi} \frac{A R^{\prime 2}-2 C R R^{\prime}+B R^{2}}{\sqrt{R^{\prime 2}+R^{2}}} d \vartheta
$$

on the set of all smooth functions $R=R(\vartheta) \geq 0$ such that

$$
\int_{0}^{\pi} R^{2} d \vartheta=1
$$

and where $A=\psi_{0}+\omega \cos ^{2} \frac{\vartheta}{2}, B=\psi_{0}+\omega \sin ^{2} \frac{\vartheta}{2}, C=\operatorname{sign}(k) \omega \cos \frac{\vartheta}{2} \sin \frac{\vartheta}{2}$. We have performed a numerical
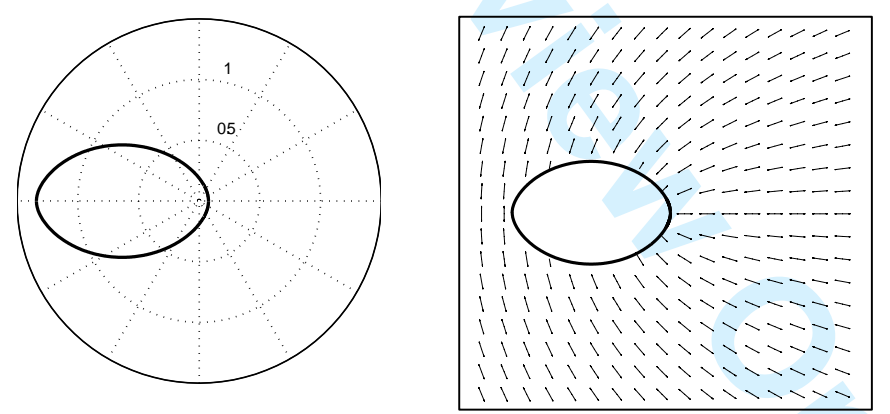

Figure 4. Left: section of a cylindrical surface minimizing the interfacial energy (33) for $k=1$. Right: core of a disclination of strength $k=1$ parallel to the axis of the cylinder. The surface-energy constants are $\psi_{0}=1$ and $\omega=0.7$. Also, $\operatorname{area}\left(D^{\prime}\right)=1$.

minimization of (33) as described in the Appendix. The results are shown in Figure 4: planar sections of disclination cores have elongated oval shapes, whose aspect ratio depends on $\omega$. As $\omega \rightarrow 0$ the planar section of the core tends to be a disk, while as $\omega$ increases the core tends to flatten along the $x$-axis (Figure $5)$.

We can use the same procedure to study the shape of the cores of a pair of disclinations located at $\boldsymbol{z}=0$ and $\boldsymbol{z}=-a \boldsymbol{i}$ with strength $k=1$ : the energy functional of the surface surrounding the disclination at 
Figure 5. Sections of disclination cores for different values of $\omega$, with $\operatorname{area}\left(D^{\prime}\right)=1$.

$\boldsymbol{z}=0$ has still the form (33), but now

$$
\begin{aligned}
& A=\psi_{0}+\frac{\omega}{2}\left(1+\frac{a \cos \vartheta+\left(\cos ^{2} \vartheta-\sin ^{2} \vartheta\right) R(\vartheta)}{\sqrt{a^{2}+R^{2}(\vartheta)+2 a R(\vartheta) \cos \vartheta}}\right) \\
& B=\psi_{0}+\frac{\omega}{2}\left(1-\frac{a \cos \vartheta+\left(\cos ^{2} \vartheta-\sin ^{2} \vartheta\right) R(\vartheta)}{\sqrt{a^{2}+R^{2}(\vartheta)+2 a R(\vartheta) \cos \vartheta}}\right) \\
& C=\frac{\omega}{2} \frac{a \sin \vartheta+2 R(\vartheta) \cos \vartheta \sin \vartheta}{\sqrt{a^{2}+R^{2}(\vartheta)+2 a R(\vartheta) \cos \vartheta}} .
\end{aligned}
$$

The results of the numerical minimization of (33) are shown in Figure 6: again sections of disclination
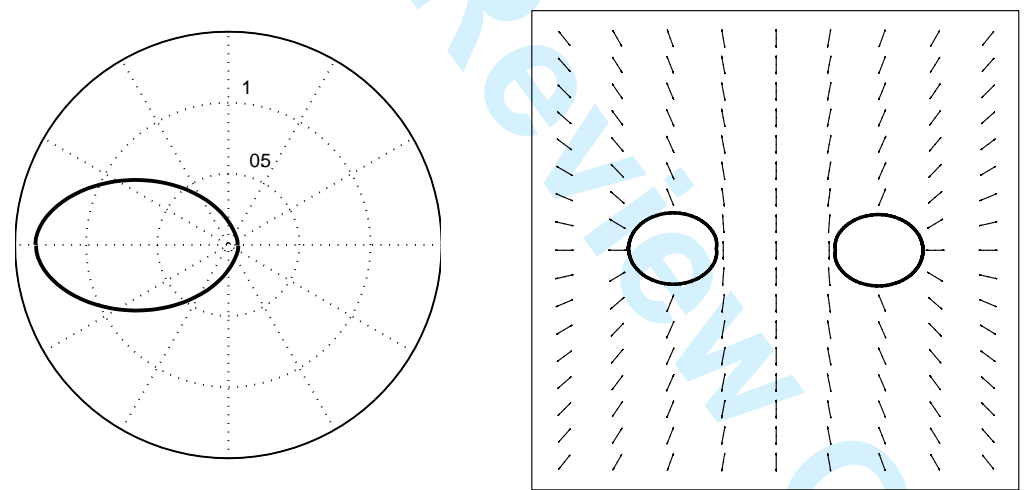

Figure 6. Left : (i) section of a cylindrical surface surrounding one of the two disclinations (the rightmost one) of a pair with strength $k=1$ and separation $a=4$, and minimizing the surface energy. Right: rescaled view of both disclination cores surrounded by the nematic field. The surface-energy constants are $\psi_{0}=1$ and $\omega=0.7$, and $\operatorname{area}\left(D^{\prime}\right)=1$.

cores have elongated oval shapes along the line joining the defecs.

\section{Applications to smectics C}

For layered structures such as smectics C, the constraint $(8)_{2}$ poses severe restrictions to the existence of solutions of the Hamilton-Jacobi equation $(8)_{1}$. Also, while for nematics the basic unknown is the surface $S$ and $f$ is just an auxiliary function of which $S$ is a level set, in smectics the function $f$ is an order parameter that should be uniquely determined by (8) and suitable boundary conditions. 
Indeed, fix a direction field $\boldsymbol{m}$ as before and consider the local coordinate system $(s, \boldsymbol{\xi})$ on $\mathbb{R}^{3}$, with $\boldsymbol{\xi}$ a coordinate system on a surface $S_{0}$ transversal to $\boldsymbol{m}$, and $s$ the arc parameter along the integral curves of $\boldsymbol{m}$, measured from the intersection of the corresponding integral curve and the initial surface $S_{0}$. In other words, $s=s(\boldsymbol{x})$ and $\boldsymbol{\xi}=\boldsymbol{\xi}(\boldsymbol{x})$ are defined so that $\boldsymbol{\xi}(\boldsymbol{x})$ is the point of intersection of the integral curve of $\boldsymbol{m}$ passing through $\boldsymbol{x}$ and the surface $S_{0}$, and $s$ is the length of the arc of this curve between $\boldsymbol{\xi}$ and $\boldsymbol{x}$.

Writing $f=\hat{f}(\boldsymbol{\xi}, s)$, it follows that $(8)_{2}$ is equivalent to

$$
\frac{\partial \hat{f}}{\partial s}=\gamma
$$

so that there exists $g=g(\boldsymbol{\xi})$ such that

$$
\hat{f}(\boldsymbol{\xi}, s)=\gamma(s+g(\boldsymbol{\xi})),
$$

and using the fact that $\boldsymbol{m} \cdot \nabla s=1,(8)_{1}$ can be rewritten as

$$
\left|\nabla_{\boldsymbol{\xi}} g\right|^{2}+\nabla s \cdot \nabla_{\boldsymbol{\xi}} g+|\nabla s|^{2}-1=\tan ^{2} \alpha .
$$

We discuss here a few special cases of (36).

Consider first a constant orientation field $\boldsymbol{m}=\boldsymbol{m}_{0}$ in a cylindrical domain $D \times \mathbb{R}$, with coordinates $(x, y, z)$ with $z$ along the axis of the cylinder. By (35) we may write the solution of (8) in the form

$$
f(x, y, z)=\gamma(z-g(x, y))
$$

and a direct calculation shows that (36) reduces to the two-dimensional eikonal equation (10) for $g$.

Assume now, to fix ideas, that the boundary condition

$$
g(x, y)=0 \quad \text { on } \partial D
$$

holds: arguing as in Section 2, we obtain a local solution (11) in the form $f(x, y, z)=\gamma(z-t(x, y) \tan \alpha)$, with $t(x, y)$ the distance function with respect to $\partial D$.

As discussed in section 2 , the distance function $t(x, y)$ is in general multivalued, with branching at the caustics, and a selection rule is necessary, for instance by requiring that $g$ be a viscosity solution of the two-dimensional eikonal equation (36) (cf. e.g., Lions [19] and Lions, Rouy and Tourin [7]). Figure 1 shows the viscosity solution corresponding to a smectic layer in a cylindrical tube with elliptic cross- section. The smectic layers are equidistant portions of conical surfaces obtained by translating the surface in Figure 1 along the vertical axis.

As a second example, consider a singular direction field $\boldsymbol{m}$ corresponding to a disclination line with strength $k=2$, as in Section 3. Specifically, let $\boldsymbol{m}=\boldsymbol{e}_{r}$, with $(r, \vartheta, z)$ cylindrical coordinates with $z$ along the disclination. Then $s=r, \boldsymbol{\xi}=(\vartheta, z)$ and $\nabla_{\boldsymbol{\xi}} g=\left(\partial_{z} g\right) \boldsymbol{e}_{z}+\frac{1}{r}\left(\partial_{\vartheta} g\right) \boldsymbol{e}_{\vartheta}$, so that (36) yields the eikonal equation on the cylinder

$$
\left(\partial_{z} g\right)^{2}+\frac{1}{r^{2}}\left(\partial_{\vartheta} g\right)^{2}=\tan ^{2} \alpha
$$

Since $g$ is independent of $r, \partial_{\vartheta} g \equiv 0$ and

$$
g=\gamma s+z \gamma \tan \alpha+\text { const. }
$$

Hence, the layers are families of equidistant half-cones with axis the disclination line.

Consider finally a hedgehog defect described by the orientation field $\boldsymbol{m}=\boldsymbol{e}_{R}$, with $(R, \vartheta, \psi)$ spherical coordinates in $\mathbb{R}^{3}$. Here $s=R, \boldsymbol{\xi}=(\vartheta, \psi)$ and $\nabla_{\boldsymbol{\xi}} g=\frac{1}{R}\left(\partial_{\vartheta} g\right) \boldsymbol{e}_{\vartheta}+\frac{1}{R \sin \vartheta}\left(\partial_{\psi} g\right) \boldsymbol{e}_{\psi}$, so that (36) yields the 
eikonal equation on the sphere

$$
\frac{1}{R^{2}}\left(\partial_{\vartheta} g\right)^{2}+\frac{1}{R^{2} \sin ^{2} \vartheta}\left(\partial_{\psi} g\right)^{2}=\tan ^{2} \alpha
$$

As before, $g$ is independent of $R$, but now this implies that the above equation has no solutions for $\alpha \neq 0$. Therefore, there is no smectic $\mathrm{C}$ configuration for which the orientation field has a hedgehog defect and the layers are constant-angle surfaces.

\section{Acknowledgments}

P.C. was supported by the Italian M.I.U.R. research grant "Modelli matematici per la scienza dei materiali" (2005).

\section{Appendix}

The numerical minimization of the surface energy functional (33) has been performed by two different techniques, which yield compatible results.

For the single-disclination problem, we have used both a modified Newton method for constrained minimization [20], and a continuous genetic algorithm [21]. For the disclination-pair problem, we have used a genetic algorithm.

To apply the modified Newton method, we may proceed as follows: first, subdivide the interval $(0, \pi)$ in $N-1$ subintervals of length $h=\pi /(N-1)$, and discretize $R$ and $R^{\prime}$ on the resulting mesh of $N$ points by centered finite differences (or, equivalently, approximate $R$ by piecewise-linear functions). Let $\boldsymbol{R}=\left(R_{1}, \ldots, R_{N}\right)$ be the vector of discretized values of $R$ : computing the integral in (33) and (34) by a Newton-Cotes method, we reduce to the problem of minimizing a function $F(\boldsymbol{R})$ subject to the constraint $c(\boldsymbol{R})=0$, where

$$
\begin{aligned}
F(\boldsymbol{R})= & \sum_{i=2}^{N-1} \frac{A_{i}\left(R_{i+1}-R_{i-1}\right)^{2}-4 h C_{i} R_{i}\left(R_{i+1}-R_{i-1}\right)+4 h^{2} B_{i} R_{i}^{2}}{2 \sqrt{\left(R_{i+1}-R_{i-1}\right)^{2}+4 h^{2} R_{i}^{2}}} \\
& +\frac{A_{1}\left(R_{2}-R_{1}\right)^{2}-2 h C_{1} R_{1}\left(R_{2}-R_{1}\right)+h^{2} B_{1} R_{1}^{2}}{2 \sqrt{\left(R_{2}-R_{1}\right)^{2}+h^{2} R_{1}^{2}}} \\
& +\frac{A_{N}\left(R_{N}-R_{N-1}\right)^{2}-2 h C_{N} R_{N}\left(R_{N}-R_{N-1}\right)+h^{2} B_{N} R_{N}^{2}}{2 \sqrt{\left(R_{N}-R_{N-1}\right)^{2}+h^{2} R_{N}^{2}}}
\end{aligned}
$$

and

$$
c(\boldsymbol{R})=h \sum_{i=2}^{N-1} R_{i}^{2}+\frac{h}{2}\left(R_{1}^{2}+R_{N}^{2}\right)-1 .
$$

Local minimizers of $F$ may be computed by a Newton method which traces the values of the Lagrange multiplier associated with the constraint, as discussed in [20]. Specifically, a sequence $\boldsymbol{R}_{(k)}$ approximating the minimizer, and a sequence $\lambda_{(k)}$ approximating the Lagrange multiplier are constructed by the iterative formulas $\boldsymbol{R}_{(k+1)}=\boldsymbol{R}_{(k)}+\boldsymbol{d}_{(k)}$ where $\lambda_{(k+1)}=\lambda_{(k)}^{Q}$, and the Newton steps $\left(\boldsymbol{d}_{(k)}, \lambda_{(k)}^{Q}\right)$ are found by solving the linear system

$$
\boldsymbol{L}_{(k)} \boldsymbol{d}_{(k)}+\boldsymbol{A}_{(k)}^{\top} \lambda_{(k)}^{Q}=-\boldsymbol{G}_{(k)}, \quad \boldsymbol{A}_{(k)} \boldsymbol{d}_{(k)}=-c_{(k)},
$$


where $\left.c_{(k)}=c\left(\boldsymbol{R}_{(k)}\right), \boldsymbol{A}_{(k)}=\nabla_{\boldsymbol{R}} c\left(\boldsymbol{R}_{(k)}\right), \boldsymbol{G}_{(k)}=\nabla_{\boldsymbol{R}} F\left(\boldsymbol{R}_{(k)}\right)\right)$, and $\boldsymbol{L}_{(k)}=\nabla_{\boldsymbol{R}}^{2} F\left(\boldsymbol{R}_{(k)}\right)+\lambda_{(k)} \nabla_{\boldsymbol{R}^{2}}^{2}\left(\boldsymbol{R}_{(k)}\right)$. The computation of the Hessian of $F$ is straightforward but cumbersome and its explicit expression will not given here.

The genetic algorithm proceeds as follows: first, restrict to the class of polynomial radial functions on $[0, \pi]$ of the form

$$
R(\vartheta)=\sum_{i=1}^{N} a_{i} \vartheta^{i-1},
$$

so that on this class the surface energy functional (33) reduces to a function $F\left(a_{1}, \ldots, a_{N}\right)$ of the $N$ coefficients $a_{i}$. This function can be evaluated numerically, for given $a_{i}$, by approximating the integral in (33) by a Newton-Cotes method. The resulting finite-dimensional minimization problem is then solved numerically in terms of the $a_{i}$, by the continuous genetic algorithm discussed in [21], by taking into account the constant-area constraint by renormalizing the coefficients at every generation.

We have checked the results obtained by the Newton method with the results obtained by the genetic algorithm, and they turned out to be consistent, in that the computed minimum surface energies differ by $0.5 \%$ after 10 iterations of the Newton method and 5000 generations of the genetic algorithm (for $\omega=0.7$ and $\left.\psi_{0}=1\right)$.

\section{References}

[1] E.G. Virga. Drops of nematic liquid crystals. Arch. Rat. Mech. Anal. 107, 371-390 (1989).

[2] E.G. Virga. Variational theories for liquid crystals. Chapman \& Hall, London (1994).

[3] P. Prinsen and P. van der Schoot. Shape and director-field transformations of tactoids. Phys. Rev. E 68, 021701 (2003).

[4] P. Prinsen and P. van der Schoot. Continuous director-field transformations of nematic tactoids. Eur. Phys. J. E 13, 35-41 (2004).

[5] P. Prinsen and P. van der Schoot. Parity breaking in nematic tactoids. J. Phys. Cond. Matter 16, 8835-8850 (2004).

[6] M.C. Calderer, C. Liu and Q. Shen. Axisymmetric configurations of bipolar liquid crystal droplets. Cont. Mech. Thermodyn. 14, 363-375 (2002).

[7] P.L. Lions, E. Rouy and A. Tourin. Shape-from-shading, viscosity solutions and edges. Numer. Math. 64, 323353 (1993).

[8] S. Osher and J.A. Sethian. Fronts propagating with curvature-dependent speed: algorithms based on Hamilton-Jacobi formulations. J. Comput. Phys. 79, 12-49, (1988).

[9] J. A. Sethian. Level set methods and fast marching methods. 2nd ed. Cambridge University Press, Cambridge (1999).

[10] V. I. Arnold. Lectures on partial differential equations. Springer-Verlag, Berlin (2004).

[11] P.G. de Gennes and J. Prost. The physics of liquid crystals. 2nd ed. Clarendon Press, Oxford (1993).

[12] J. Eells and J.H. Sampson. Harmonic mappings of Riemannian manifolds. Amer. J. Math., 86 109-160, (1964).

[13] A. Rapini and M. Papoular, Distorsion d'une lamelle nématique sous champ magnétique. Conditions d'ancrage aux parois. Journal de Physique Colloque C4, C4-54-C4-46 (1969).

[14] R. Rosso and M.C.P. Brunelli. Forces on nematic disclinations with optimal core. Continuum Mech. Thermodyn. 13,383-398 (2001).

[15] P. Biscari and M.C. Calderer. Telephone-cord instabilities in thin smectic capillaries. Phys. Rev. E 71051701 (2005).

[16] K.J. Kidney, G. McKay and I.W. Stewart. Energy considerations for parabolic cyclides in SmA liquid crystals. Mol. Cryst. Liq. Cryst, 438 1827-1833 (2005).

[17] G. McKay and F.M. Leslie. A continuum theory for smectic liquid crystals allowing layer dilation and compression. Euro. Jnl. of Applied Mathematics, 8 273-280 (1997).

[18] G. McKay. Spherical, cylindrical and toroidal layering of smectic C liquid crystals. Mol. Cryst. Liq. Cryst., 366 403-412, (2001).

[19] P.L. Lions. Generalized Solutions of Hamilton-Jacobi Equations. Research Notes in Mathematics 69, Pitman, Boston (1982).

[20] J.F. Bonnans, J.C. Gilbert, C. Lemaréchal, C.A. Sagastizábal. Numerical optimization. Springer-Verlag, Berlin (2003).

[21] R.L. Haupt and S.E. Haupt. Practical genetic algorithms. 2nd ed. Wiley, Oxford (2004). 